\title{
Coenzyme Q10 improves the survival and reduces inflammatory markers in septic patients
}

\author{
Soltani $\mathrm{R}^{1}$, Alikiaie $\mathrm{B}^{2}$, Shafiee $\mathrm{F}^{3}$, Amiri $\mathrm{H}^{4}$, Mousavi $\mathrm{S}^{1}$ \\ Department of Clinical Pharmacy and Pharmacy Practice, School of Pharmacy and Pharmaceutical \\ Sciences, Isfahan University of Medical Sciences, Isfahan, Iran. s.mousavi@pharm.mui.ac.ir
}

\begin{abstract}
OBJECTIVE: This study aimed to evaluate the effect of Coenzyme Q10 (CoQ10) administration to patients in the early phase of sepsis to determine its effect on the markers of inflammation and the clinical outcomes of septic patients.

BACKGROUND: Previous studies showed that CoQ10 levels were decreased in septic patients and worsening of mitochondrial dysfunction was observed.

METHODS: In this randomized controlled trial septic patients $(n=40)$ received $100 \mathrm{mg}$ CoQ10 twice a day for seven days added to standard treatment of sepsis. As a primary endpoint levels of Interleukin 6 (IL-6), Tumor Necrosis Factor- $\alpha$ (TNF- $\alpha$ ), Glutathione peroxidase and malondialdehyde (MDA) were assessed at baseline, third and 7th day after the intervention. Secondary endpoints included assessment of clinical scores and in-hospital mortality.

RESULTS: There was no difference in baseline inflammatory and oxidative injury markers between the groups. TNF- $\alpha$ and MDA levels decreased significantly in the CoQ10 group on the 7th day of the study (P:0.003 for both). There was a significant difference in the in-hospital mortality in the CoQ10 group compared to the control group (P:0.01).

CONCLUSION: These findings suggest that CoQ10 has a positive effect on clinical parameters as well as mitochondrial dysfunction when administered in the early phase of sepsis (Tab. 2, Fig. 1, Ref. 38). Text in PDF www.elis.sk.

KEY WORDS: coenzyme Q10, sepsis, mitochondria, antioxidants.
\end{abstract}

\section{Introduction}

Sepsis is a systemic inflammatory response to an infection leading to endothelial dysfunction, impairment of microcirculation, tissue hypoxia, apoptosis and finally multiple organ dysfunction and death (1). Despite the development of new strategies and antibiotics, the overall mortality rate of sepsis is still high and has not been reduced significantly over the last decade (2, 3). During sepsis, the inflammatory response caused by excessive secretion of pro-inflammatory mediators, such as Tumor Necrosis Factor- $\alpha$ (TNF- $\alpha$ ), and reactive oxygen species (ROS) mainly induced by

${ }^{1}$ Department of Clinical Pharmacy and Pharmacy Practice, School of Pharmacy and Pharmaceutical Sciences, Isfahan University of Medical Sciences, Isfahan, Iran, ${ }^{2}$ Department of Anesthesiology and Intensive Care, Alzahra hospital, Isfahan University of Medical Sciences, Isfahan, Iran, ${ }^{3}$ Department of Biotechnology, School of Pharmacy and Pharmaceutical Sciences, Isfahan University of Medical Sciences, Isfahan, Iran, and ${ }^{4}$ Pharm D student, School of Pharmacy and Pharmaceutical Sciences, Isfahan University of Medical Sciences, Isfahan, Iran

Address for correspondence: S. Mousavi, Dr, Department of Clinical Pharmacy and Pharmacy Practice, School of Pharmacy and Pharmaceutical Sciences, Isfahan University of Medical Sciences, HezarJerib Avenue, Isfahan 81746-73461, Iran.

Phone: +983137927072 , Fax: +983136680011 .

Acknowledgement: This study was granted by the research deputy of Isfahan University of Medical Sciences (grant number:193114). bacterial endotoxin, induce changes in cellular metabolism, particularly mitochondrial dysfunction (4-6). Immune cells use ROS in order to support their functions and therefore need adequate levels of antioxidant defenses in order to avoid the harmful effect of an excessive production of ROS. The endogenous antioxidant systems themselves can also be affected by oxidative stress via oxidation and peroxidation of the component enzymes. Oxidative stress mediated damage to mitochondrial DNA can lead to a cycle of ROS production and further mitochondrial damage. Also, the antioxidant system becomes completely overwhelmed and eventually cell death occurs, leading to multiple organ dysfunction (7-9). Recent reviews emphasize the importance of mitochondrialtargeted antioxidants in sepsis (10-12).

Coenzyme Q10 (CoQ10), also known as ubiquinone, plays a critical role in the mitochondrial respiratory chain for ATP production. CoQ10 is a lipophilic mobile electron carrier that is located in the inner mitochondrial membrane. The mitochondrial electron transport chain contains two transporters (CoQ10 and cytochrome C) and four complexes (I-IV) (13). CoQ10 has been reported to have the ability to act as an effective antioxidant. CoQ10 prevents peroxidation damage to cell membranes, regenerates $\alpha$-tocopherol, maintains thiol levels and controls mitochondrial transition pore opening which is involved in apoptosis (14-16).

CoQ10 is primarily synthesized endogenously in the endoplasmic reticulum; however, a small portion of CoQ10 may be 
obtained from dietary sources. Therefore, low CoQ10 levels may result from impairment in CoQ10 synthesis, decreased dietary intake or increased requirement such as states of increased oxidative stress (17). Previous studies have shown that CoQ10 levels decrease in septic patients compared to healthy subjects (18). Also, CoQ10 levels were inversely associated with levels of vascular endothelial markers and inflammatory molecules. In many studies, the levels of pro-inflammatory cytokines such as TNF- $\alpha$ and Interleukin-6 (IL-6) have been shown to decrease significantly following CoQ10 supplementation (19-21). So, if there is a deficit in CoQ10 during sepsis, CoQ10) could be therapeutically used in the prevention and treatment of sepsis associated multi organ failure to boost mitochondrial function and to mitigate cellular damage caused by oxidative stress. Some animal models have been used to test the impact of CoQ10 on the consequences of sepsis. Injection of CoQ10 into the rostral ventrolateral medulla (medullary origin of sympathetic vasomotor tone) of rats diminished mortality and lipopolysaccharide-induced hypotension during exposure to lipopolysaccharide (22). Doninno et al (23), in a pilot trial, showed that administration of exogenous Ubiquinol (the reduced form of CoQ10) could increase plasma CoQ10 levels and improve mitochondrial function in patients with severe sepsis or septic shock. In one hand, this result is interesting because it is the first report of low CoQ10 levels in patients with septic shock. On the other hand, the main limitation of this study is that it is a post hoc analysis of a prospective randomized trial of simvastatin versus placebo in patients with septic shock. However, as mentioned by the authors, the sample size for this study is small and larger prospective observational trials measuring CoQ10 in patients with septic shock are required. But do these low CoQ10 levels imply CoQ10 supplementation in patients with sepsis? This study aimed to evaluate the effects of CoQ10 supplementation in patients in early stage of sepsis to determine its effects on markers of inflammation and oxidative injury as well as the clinical outcome of septic patients.

\section{Methods}

A prospective, randomized controlled trial was undertaken in a single center from September 2016 to March 2017. The study population consisted of consecutive patients admitted to the Intensive Care Units (ICU) of Alzahra hospital, a tertiary-level university affiliated hospital. The ethic committee of University of Medical Sciences approved the study. Informed consent was obtained from patients or next of kin or appropriate surrogate before participation in the study. The study was registered with the Registry of Clinical Trials (IRCT201504051497N4).

During the study period, all patients admitted to the ICU were screened for study eligibility and randomly assigned to one of the groups. Inclusion criteria were: age $\geq 18$ years; having two or more of the four systemic inflammatory syndrome (SIRS) criteria (24) (defined as the following conditions: heart rate $>90$ beat $/ \mathrm{min}$, respiratory rate $>20$ breath $/ \mathrm{min}$ or $\mathrm{PaCO} 2<32 \mathrm{mmHg}$, white blood cells $(\mathrm{WBC})>12,000 \mathrm{cell} / \mathrm{mm}^{3}$, or $<4000 \mathrm{cell} / \mathrm{mm}^{3}$, or $>$ $10 \%$ immature [band] cells): suspected or confirmed infection (pneumonia, peritonitis, pyelonephritis and so on): and APACHE II (acute physiology and chronic health evaluation) score more than 15 at the admission.

Exclusion criteria were coagulopathy (International Normalized Ratio [INR] > 2), current use of CoQ10 or any other antioxidant, inability to receive enteral medication, pregnancy, current participation in other studies, and end-stage or DNR (do not resuscitate) patients.

Eligible patients were enrolled, received CoQ10 100mg twice daily for 7 days. The dose was chosen based on the previous trial which showed that a dose of $200 \mathrm{mg}$ was well-tolerated in septic patients. Patients in the control group received standard treatment for sepsis without supplementation with CoQ10 or other antioxidants. According to the latest international guidelines $(24,25)$, standard treatments for sepsis were implemented as follows for both groups: early resuscitation within the first 6 hours of admission, appropriate diagnostic studies to ascertain causative organisms, early administration of broad-spectrum antibiotic therapy and reassessment of antibiotic therapy with microbiologic tests and clinical data to narrow the coverage.

Venous blood was collected before administration of study drug (day 1) and at 3rd and 7 th days thereafter. Samples were centrifuged at $1500 \mathrm{rpm}$ for 15 minutes to remove cells and cellular debris. The plasma was stored at $-80{ }^{\circ} \mathrm{C}$ until the time of analysis. Levels of IL-6, TNF- $\alpha$, and glutathione peroxidase were measured by commercially available enzyme-linked immunosorbent assay (ELISA) kits (Zell Bio and IBL international, Germany) and malondialdehyde (MDA) level was determined by spectrophotometric analysis kit (ZellBio, Germany) according to manufacturer's instructions.

Upon enrollment, we recorded demographic data, admission diagnosis, vital signs, clinical laboratory values, and calculated the APACHE II score. Vital signs and laboratory values were also collected at all subsequent time points and outcome data, including length of ICU stay, length of hospital stay and mortality were collected at patient discharge.

Sequential organ failure assessment score (SOFA score) and Simplified Acute Physiology Score (SAPS) II were calculated at studied time point.

The primary endpoint of the study was assessment of inflammation via IL- 6 and TNF- $\alpha$ measurements and assessment of changes in oxidative injury via glutathione peroxidase and MDA.

The secondary endpoints were assessment of clinical outcomes, including SOFA and SAPS II scores and length of ICU stay and in-hospital mortality.

According to the systematic review by Zhai et al (21), CoQ10 supplementation significantly decreased TNF- $\alpha$ level by $0.45 \mathrm{pg} /$ $\mathrm{ml}\left[\mathrm{MD}=-0.45,95 \% \mathrm{CI}(-0.67\right.$ to -0.24$\left.) \mathrm{pg} / \mathrm{ml}, \mathrm{I}^{2}=0 \%\right]$. Assuming an alpha of 0.05 , a power of 0.80 and a standard deviation of $0.6(26)$, with a drop-out rate of $10 \%$, we estimated a sample size of 30 patients in each arm of the study. The formula used was as follows:

$$
\mathrm{n}=\left[\left(\mathrm{Z}_{\alpha / 2}+\mathrm{Z}_{\beta}\right)^{2} \times\left\{2(\mathrm{o})^{2}\right\}\right] /(\mu 1-\mu 2)^{2}
$$




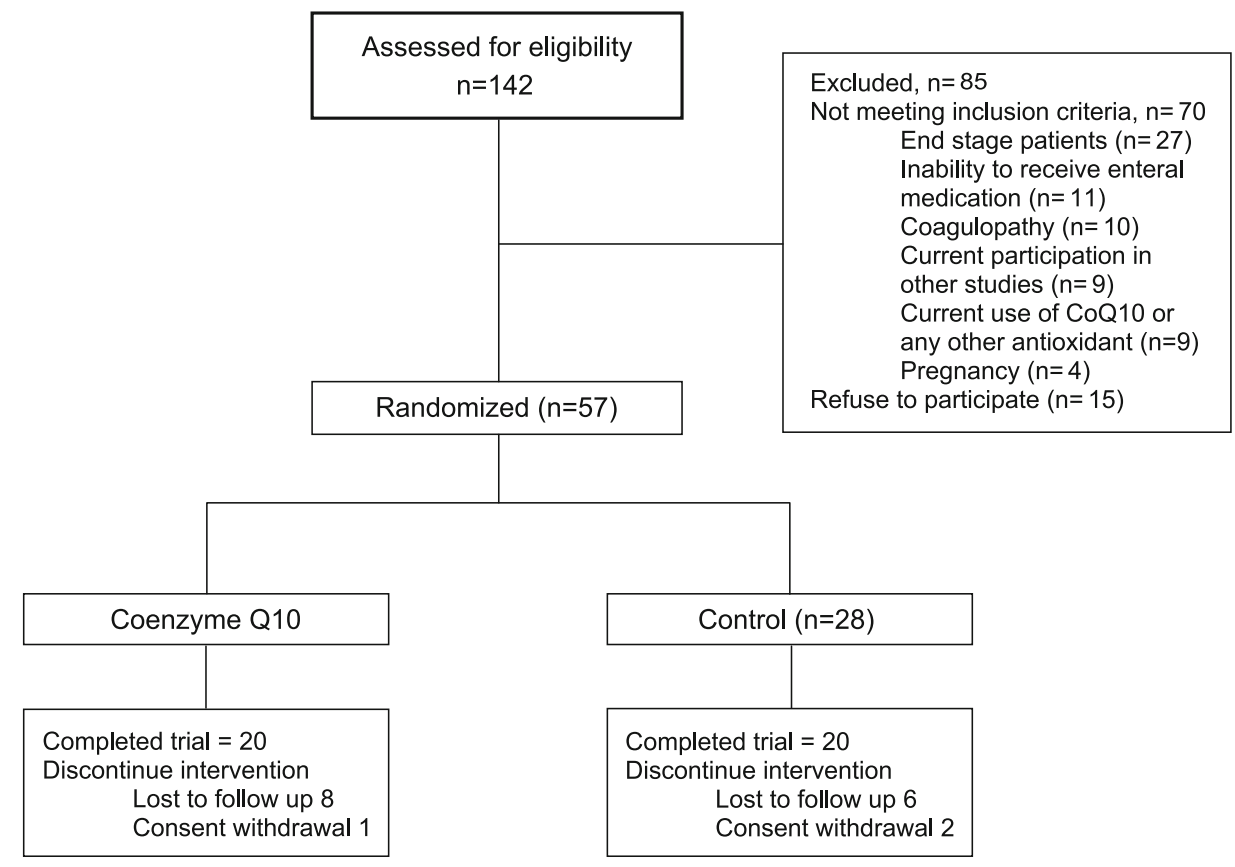

Fig. 1. Progress through the stages of the trial.

\section{Statistical analysis}

The baseline characteristics and laboratory data presented as the means and standard deviations (SD) for continuous variables and as frequencies and percentages for categorical variables. Analysis of variance (ANOVA) and analysis of covariance (ANCOVA) were used for comparison of values between the groups in each day with later test being used for control of the effects of baseline values. Fisher's exact test was used for comparison of clinical outcome between the groups. A value of $p \leq 0.05$ was considered statistically significant. All analyses were performed using SPSS statistics software V23.0 (SPSS Inc; Chicago, IL, USA).

\section{Results}

During the study period, 142 consecutive patients admitted to the ICU were screened and 40 met criteria for enrollment. Figure 1 reports the trial implementation profile according to the consolidated standard of reporting trials. Of 142 patients who were assessed for eligibility, 57 were randomly assigned (29 patients to Co Q10 and 28 patients to Control group) with a ratio of 1:1. Twenty patients in each group completed the protocol. Reasons for interrupting treatment are reported in Figure 1. For eight patients in the intervention group and four patients in the control group, the blood samples couldn't be obtained at the measurement time of the study and so these patients were considered as lost to follow up.

The baseline characteristics including age, gender, admission diagnosis and APACHE II score $(\mathrm{p}>0.05)$ were similar in both groups (Tab. 1).

There was no difference in baseline inflammatory and oxidative markers between the groups. IL- 6 levels decreased in the CoQ10 group on the third and the 7th day of the study as com- pared to the control group and baseline, but not significantly (p: 0.22 ). TNF- $\alpha$, and MDA levels decreased significantly (p: 0.00 and p: 0.003 , respectively) in the CoQ10 group on the 7 th day of the study compared to the control group and baseline. There was no difference between the CoQ10 and the control groups in the levels of glutathione peroxidase over time (: 0.1) (Tab. 2).

There was no difference in ICU length of stay $(42.5 \pm$ 32.4 in CoQ10 group versus $39.5 \pm 24.2$ in the control group; p: 0.74). There was a significant difference in the in-hospital mortality between patients receiving CoQ10 versus control group (4 (20\%) versus 13 (655); p: 0.01). Compared to controls, CoQ10 was not effective in improving SOFA (p: 0.55) and SAPS II (p: 0.7) scores during the study period (Tab. 2).

Tab. 1. Baseline and patient characteristics.

\begin{tabular}{lcc}
\hline Variables & $\begin{array}{c}\text { Co Q10 group } \\
\mathrm{n}=20\end{array}$ & $\begin{array}{c}\text { Control group } \\
\mathrm{n}=20\end{array}$ \\
\hline Age in years, mean \pm SD & $47.8 \pm 22$ & $55.6 \pm 19.4$ \\
\hline Males, number (percentage) & $17(85)$ & $14(70)$ \\
\hline APACHE II score, mean \pm SD & $15.3 \pm 5.2$ & $16.4 \pm 6.4$ \\
\hline ICU length of stay, mean \pm SD & $42.5 \pm 32.4$ & $39.5 \pm 24.2$ \\
\hline Mortality, number (percentage) & $4(20)$ & $13(65)$ \\
\hline Admission diagnosis, number (percentage) & & \\
Multiple Trauma & $6(30)$ & $4(20)$ \\
ARDS & $1(5)$ & $5(25)$ \\
ICH & $3(15)$ & $3(15)$ \\
Pneumonia & $2(10)$ & $0(0($ \\
Hydrocephalus & $0(0)$ & $2(10)$ \\
Post-operative & $4(20)$ & $1(5)$ \\
Pancreatitis & $0(0)$ & $2(10)$ \\
Other & $4(20)$ & $3(15)$ \\
\hline ARDS - acute respiratory distress syndrome, $\mathrm{ICH}-$ intracerebral hemorrhage
\end{tabular}




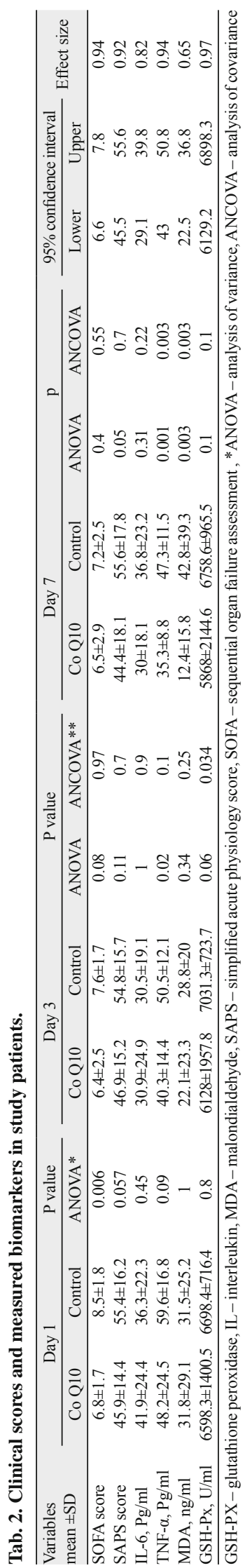

\section{Discussion}

The current study has shown that CoQ10 supplementation at the early stage of sepsis diagnosis and as addition to standard treatment of sepsis could reduce the levels of inflammatory and oxidative markers as well as mortality rates in septic patients.

As mentioned earlier, several studies have demonstrated that CoQ10 levels are low during sepsis $(18,27,28)$; CoQ10 plays an essential role in the electron transport chain as the carrier of electrons from complexes I and II to complex III. Disruption of this mechanism can compromise oxidative phosphorylation in the mitochondria, thereby leading to decreased levels of cellular ATP production. So, CoQ10 supplementation could be used in the prevention and treatment of sepsisassociated multiple organ failure to boost mitochondrial function and to mitigate cellular damage caused by oxidative stress. Multiple rat models have shown beneficial impact of CoQ10 in sepsis and have found decreased oxidative stress, prevention of mitochondrial damage, less renal and acute liver dysfunction, and neuroprotective effects $(12,29,30)$. Donnino et al., conducted two studies. In the first (18) they provided original data suggesting a CoQ10 deficiency in patients with septic shock. In the second study (23) which was a randomized, double-blind, placebo-controlled, pilot trial, they administered $200 \mathrm{mg}$ of enteral ubiquinol or placebo twice daily for up to seven days to 38 patients with severe sepsis or septic shock. The results showed that plasma CoQ10 levels increased in patients who received ubiquinol. The other markers including vascular endothelial function, mitochondrial dysfunction, and inflammation revealed no statistically significant difference between the ubiquinol and the placebo groups. The clinical outcomes were not evaluated in this study.

Following the initial host and microbial interaction in the sepsis process, inflammatory cytokines (such as TNF- $\alpha$, IL- 6 and IL-8) are released by activating macrophages and CD4 T cells within the first hour after infection. To prevent severe damage, anti-inflammatory mediators (such as IL-10) and antioxidants are released. The endogenous antioxidants become overwhelmed and the vicious cycle of oxidant production leads to progression of sepsis to severe sepsis, multiple organ failure, and septic shock. Therefore, intervention to potentiate the endogenous antioxidants and mitigate oxidative stress at early stage of sepsis may play a significant role in the prevention of multi- organ dysfunction and disease progression in sepsis $(9,31,32)$. In this study, CoQ10 supplementation at early phase of sepsis significantly reduced inflammatory markers in patients who received CoQ10. The mortality rates were reduced in these patients as well. We should consider that CoQ10 was added as an adjunct tto standard treatment of sepsis in our study. Therefore, the lowest mortality rate in the CoQ10 group compared to the control group could be a result of addition of CoQ10; however, our sample size was small and our study was not placebo-controlled, so we should interpret this result cautiously. The two clinical scores (SOFA and SAPS) didn't change significantly between groups, although, slightly decreased in the CoQ10 group.

One of the main problems in sepsis are oxygen extraction and consumption. Many studies focused on macrocirculatory resuscitation (means aggressive management with fluids, blood transfusion and inotropic agents) in the earliest stage of sepsis, but still demonstrate multisystem organ dysfunction and shock (33-35). The recent researches in sepsis are focusing on two separate mechanism. The first is the role of the microcirculation in the body and the mixed response of various circulatory beds to sepsis (36-38). The second is that the cells and mitochondria receiving adequate perfusion and oxygenation may still have a decreased ability to properly utilize energy to form ATP, referred to as cytopenic hypoxia, which can lead to profound metabolic alterations and the ability to supply energy to the tissues. Therefore, improving tissue oxygenation through disruption of the mechanisms that comprise oxidative phosphorylation in the mitochondria, could be a possible mechanism of CoQ10 in the reduction of inflammatory markers and mortality in septic patients.

Fortunately, we didn't observe any adverse effect from CoQ10 supplementation in this study and it could be a safe adjunct to other standard treatments of sepsis.

The duration of therapy was relatively short (maximum of seven days), and a longer duration and follow-up period may be needed, especially for long-term patient-centered outcomes.

\section{Conclusion}

In conclusion, despite some limitations of this study, especially the small sample size, CoQ10 supplementation shows positive effects on clinical outcomes of patients with sepsis, when administered at early phase of sepsis and as an adjunct to standard treatment 
of sepsis. This study is a new step towards other studies, testing CoQ10 as a potential agent for patients at early phase of sepsis.

\section{References}

1. Chong J, Dumont T, Francis-Frank L, Balaan M. Sepsis and septic shock: a review. Crit Care Nurs Q 2015; 38 (2): 111-120.

2. Fleischmann C, Scherag A, Adhikari NK et al. Assessment of global incidence and mortality of hospital-treated sepsis. Current estimates and limitations. Am J Respir Crit Care Med 2016; 193 (3): 259-272.

3. Liu V, Escobar GJ, Greene JD et al. Hospital deaths in patients with sepsis from 2 independent cohorts. JAMA 2014; 312 (1): 90-92.

4. Duran-Bedolla J, de Oca-Sandoval MAM, Saldaña-Navor V, Villalobos-Silva JA. Sepsis, mitochondrial failure and multiple organ dysfunction. Clin Invest Med 2014; 37 (2): E58-E69.

5. Jeger V, Djafarzadeh S, Jakob SM, Takala J. Mitochondrial function in sepsis. Eur J Clin Invest. 2013; 43 (5): 532-542.

6. Singer $M$. The role of mitochondrial dysfunction in sepsis-induced multiorgan failure. Virulence 2014; 5 (1): 66-72.

7. Bavunoglu I, Genc H, Konukoglu D et al. Oxidative stress parameters and inflammatory and immune mediators as markers of the severity of sepsis. J Infect Dev Ctries 2016; 10 (10): 1045-1052.

8. Galley HF. Oxidative stress and mitochondrial dysfunction in sepsis. $\mathrm{Br}$ J Anaesth 2011; 107 (1): 57-64.

9. Prauchner CA. Oxidative stress in sepsis: pathophysiological implications justifying antioxidant co-therapy. Burns 2017; 43 (3): 471-485.

10. Dare AJ, Phillips AR, Hickey AJ et al. A systematic review of experimental treatments for mitochondrial dysfunction in sepsis and multiple organ dysfunction syndrome. Free Radic Biol Med 2009; 47 (11): 1517-1525.

11. Zakharova VV, Pletjushkina OY, Zinovkin RA, Popova EN, Chernyak BV. Mitochondria-Targeted Antioxidants and Uncouplers of Oxidative Phosphorylation in Treatment of the Systemic Inflammatory Response Syndrome (SIRS). J Cell Physiol 2017; 232 (5): 904-912.

12. Zheng G, Lyu J, Huang $J$ et al. Experimental treatments for mitochondrial dysfunction in sepsis: A narrative review. J Res Med Sci 2015; 20 (2): 185-195.

13. Bhagavan HN, Chopra RK. Coenzyme Q10: absorption, tissue uptake, metabolism and pharmacokinetics. Free Radic Res 2006; 40 (5): 445-453.

14. Littarru GP, Tiano L. Clinical aspects of coenzyme Q10: an update. Nutrition 2010; 26 (3): 250-254.

15. Emmanuele V, López LC, Berardo A et al. Heterogeneity of coenzyme Q10 deficiency: patient study and literature review. Arch Neurol 2012; 69 (8): 978-983.

16. Quinzii CM, Hirano M. Coenzyme Q10 and mitochondrial disease. Dev Disabil Res Rev2010; 16 (2): 183-188.

17. Abitagaoglu S, Akinci SB, Saricaoglu F et al. Effect of coenzyme Q10 on organ damage in sepsis. Bratisl Med J 2015; 116 (7): 433-439.

18. Donnino MW, Cocchi MN, Salciccioli JD et al. Coenzyme Q10 levels are low and may be associated with the inflammatory cascade in septic shock. Crit Care 2011; 15 (4): R189.

19. Fan L, Feng Y, Chen GC et al. Effects of coenzyme Q10 supplementation on inflammatory markers: a systematic review and meta-analysis of randomized controlled trials. Pharmacol Res. 2017; 119: 128-136.

20. Farsi F, Heshmati J, Janani L et al. Can coenzyme Q10 supplementation effectively reduce human tumour necrosis factor- $\alpha$ and interleukin- 6 levels in chronic diseases? Protocol for a systematic review and meta-analysis of randomised controlled trials. BMJ Open 2017; 7 (10): e016841.
21. Zhai J, Bo Y, Lu Y, Liu C, Zhang L. Effects of coenzyme Q10 on markers of inflammation: a systematic review and meta-analysis. PloS One 2017; 12 (1): e0170172.

22. Chuang YC, Chan JY, Chang AY et al. Neuroprotective eff ects of coenzyme Q10 at rostral ventrolateral medulla against fatality during experimental endotoxemia in the rat. Shock 2003; 19: 427-432.

23. Donnino MW, Mortensen SJ, Andersen LW et al. Ubiquinol (reduced Coenzyme Q10) in patients with severe sepsis or septic shock: a randomized, double-blind, placebo-controlled, pilot trial. Crit Care 2015; 19 (1): 275.

24. Seymour CW, Liu VX, Iwashyna TJ et al. Assessment of clinical criteria for sepsis: for the Third International Consensus Definitions for Sepsis and Septic Shock (Sepsis-3). JAMA. 2016; 315 (8): 762-774

25. Rhodes A, Evans LE, Alhazzani W et al. Surviving sepsis campaign: international guidelines for management of sepsis and septic shock: 2016. Intens Care Med 2017; 43 (3): 304-377.

26. Farsi F, Mohammadshahi M, Alavinejad $P$ et al. Functions of Coenzyme Q10 Supplementation on Liver Enzymes, Markers of Systemic Inflammation, and Adipokines in Patients Affected by Nonalcoholic Fatty Liver Disease: A Double-Blind, Placebo-Controlled, Randomized Clinical Trial. J Am Coll Nutr 2016; 35 (4): 346-353.

27. Dupic L, Huet O, Duranteau J. Coenzyme Q 10 deficiency in septic shock patients. Crit Care 2011; 15 (5): 194.

28. Coppadoro A, Berra L, Kumar A et al. Critical illness is associated with decreased plasma levels of coenzyme Q10: a cross-sectional study. J Crit Care 2013; 28 (5): 571-576.

29. Neyrinck AM, Catry E, Sohet FM et al. Lack of anti-inflammatory effect of coenzyme Q10 supplementation in the liver of rodents after lipopolysaccharide challenge. Clinical Nutrition Experimental 2015; 1: 10-18.

30. Ozer EK, Goktas MT, Kilinc I et al. Coenzyme Q10 improves the survival, mesenteric perfusion, organs and vessel functions in septic rats. Biomed Pharmacother 2017; 91: 912-919.

31. McCreath G, Scullion M, Lowes D, Webster N, Galley H. Pharmacological activation of endogenous protective pathways against oxidative stress under conditions of sepsis. Br J Anaesth 2015; 116 (1): 131-139.

32. Mantzarlis K, Tsolaki V, Zakynthinos E. Role of Oxidative Stress and Mitochondrial Dysfunction in Sepsis and Potential Therapies. Oxid Med Cell Longev 2017; 2017: 5985209.

33. Head LW, Coopersmith CM. Evolution of Sepsis Management: From Early Goal-Directed Therapy to Personalized Care. Adv Surg 2016; 50 (1): 221-234.

34. Vincent JL. Current management of sepsis and septic shock. Signa Vitae 2016; 11 (2): 14-16.

35. Andrews B, Semler MW, Muchemwa L et al. Effect of an early resuscitation protocol on in-hospital mortality among adults with sepsis and hypotension: a randomized clinical trial. JAMA 2017;318 (13): 1233-1240.

36. Ince C, Mik EG. Microcirculatory and mitochondrial hypoxia in sepsis, shock, and resuscitation. J Appl Physiol 2015; 120 (2): 226-235.

37. van der Voort PH, van Zanten M, Bosman RJ et al. Testing a conceptual model on early opening of the microcirculation in severe sepsis and septic shock: a randomised controlled pilot study. Eur J Anaesthesiol 2015; 32 (3): 189-198.

38. Colbert JF, Schmidt EP. Endothelial and microcirculatory function and dysfunction in sepsis. Clin Chest Med 2016; 37 (2): 263-275.

Received September 8, 2019. Accepted November, 2019. 